\title{
Pull-Through Buddy Wire Technique for Endovascular Thrombectomy in Patients with Acute Ischemic Stroke: Technical Note
}

\author{
Pin-Yi Chiang, MD, Yen-Heng Lin, MD, MS, Yu-Cheng Huang, MD, Chung-Wei Lee, MD, PhD \\ Department of Medical Imaging, National Taiwan University Hospital, Taipei, Taiwan
}

Excessive tortuosity is a notable cause of failed endovascular thrombectomy for acute large-vessel occlusion stroke. Transcervical access (TCA) is a commonly proposed solution for overcoming this difficulty. However, the large-bore catheter usually used in TCA increases the risk of serious local complications. This paper presents a modified technique for TCA that uses a pull-through buddy wire (PTBW) to track a large-bore femoral guiding sheath (GS) into the carotid artery via a small carotid puncture site. The carotid puncture site can be easily managed through gentle manual compression. Two illustrative cases using this technique to deal with a large aortic arch and tortuous left common carotid artery are reported. In both cases, recanalization was achieved after successful GS placement. Using a PTBW is feasible in TCA.

Key Words: Intracranial embolism; Thrombectomy; Endovascular procedures; Stroke

\section{INTRODUCTION}

Endovascular thrombectomy (EVT) is a standard treatment for acute large-vessel occlusion (LVO) stroke. ${ }^{1,2}$ Rapid and optimal recanalization is the treatment goal, but technical success is not always achieved. Unfavorable anatomy is the most common reason for failed thrombectomies. ${ }^{3}$ Difficult anatomy, such as an elongated aortic arch or severely calcified, tortuous, or stenotic proximal cervical vasculature, may prevent the operator from establishing a stable position for the guiding catheter and subsequent fertile treatment. Transradial, transbrachial, transcervical, and transvertebral routes have been proposed as alternatives to the transfemoral approach.
Among these, transcervical access (TCA) has been described in the literature for overcoming the difficult anatomy of extracranial neurovascular vessels. ${ }^{4-8}$ Nevertheless, the reported complication rate is higher than that for the transfemoral approach, because it requires more experience to perform and better understanding of the vascular anatomy. $5,7,9$ In this paper, we present a modified TCA using a pull-through buddy wire technique (PBWT) for anterior circulation LVO stroke. Two illustrative cases are reported.

\section{Correspondence to:} Chung-Wei Lee, MD, PhD

Department of Medical Imaging, National Taiwan University Hospital, No. 7, Chung-Shan South Rd, Taipei 100, Taiwan

Tel: +886-2-23123456

Fax: +886-2-23224552

E-mail: rad.chungweilee@gmail.com

Received: November 13, 2020 Revised: December 15, 2020 Accepted: December 30, 2020

Copyright $\odot 2021$ Korean Society of Interventional Neuroradiology

This is an Open Access article distributed under the terms of the Creative Commons Attribution Non-Commercial License (http://creativecommons.org/licenses/by-nc/4.0) which permits unrestricted non-commercial use, distribution, and reproduction in any medium, provided the original work is properly cited.

pISSN 2093-9043 eISSN 2233-6273 


\section{TECHNIQUES WITH CASE DESCRIPTION}

\section{Pull-through buddy wire technique}

Under local anesthesia, an 8-Fr Flexor Shuttle guiding sheath (GS) (COOK Medical, Bloomington, IN, USA) is first inserted into the proximal descending aorta through the transfemoral approach. Under ultrasonographic guidance, a $20-\mathrm{G}$ venous catheter is retrogradely punctured into the distal common carotid artery (CCA), followed by insertion of a 0.014 -inch microwire. A snare catheter is inserted into the aortic arch through the femoral GS. The microwire is then captured by the snare catheter (Fig. 1A) and pulled out through the GS. Both ends of the microwire are held firmly with torque devices, allowing it to serve as a fixed buddy wire. On the femoral side, the microwire is tensed and the GS is tracked into distal CCA (Fig. 1B). After successful GS placement, the microwire is kept locked to provide stability, and a reperfusion catheter can be used parallel to the microwire through the GS.

\section{Case 1}

An 87-year-old female with a medical history of hypertension and dyslipidemia presented to our emergency department with severe dysarthria, slurred speech, and right hemiplegia for 1.5 hours. A National Institutes of Health Stroke Scale (NIHSS) score of 18 was reported. Computed tomography angiography (CTA) revealed the middle cerebral artery (MCA)

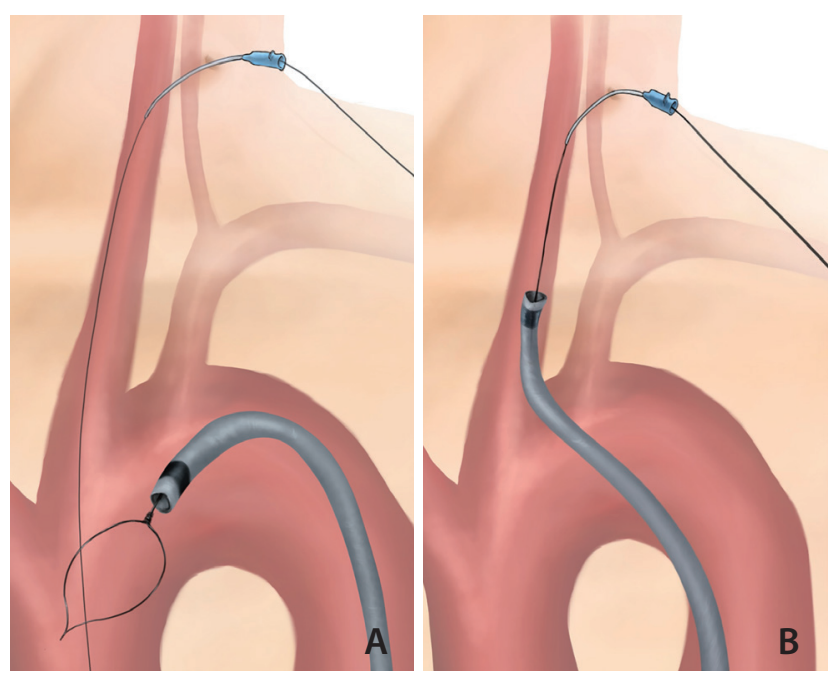

Fig. 1. Illustration of the pull-through technique in the CCA. (A) A microwire through the puncture route in the common carotid artery goes down to the ascending aorta. The snare through the guiding catheter from the transfemoral approach catches the microwire. (B) The guiding catheter can be guided along the tensed microwire. occlusion at the M1 segment. The patient was transferred to a neuroangiography suite for endovascular treatment.

Excessive tortuosity of the aorta was reviewed using CTA before the procedure was performed (Fig. 2A). Reaching through the aorta through the transfemoral approach was considered difficult because of a low-lying, tortuous left CCA and the fact that the aorta was type III; therefore, PBWT was chosen. After successful placement of a GS into the distal left CCA (Fig. 2B, C), a 6-Fr reperfusion catheter (ACE 60; Penumbra, Alameda, CA, USA) was then inserted into the GS. Suction thrombectomy was performed with successful thrombolysis in cerebral infarction (TICl) 3 recanalization in 1 pass (Fig. 2D, E). The procedural time was 20 minutes from femoral puncture to guiding catheter placement inside CCA, 8 minutes for carotid puncture, and the total time taken to complete recanalization was 37 minutes from femoral puncture. After recanalization, the femoral puncture site was closed with a vascular closure device (VCD) (Angioseal VIP, $8 \mathrm{Fr}$; Terumo, St. Jude Medical, St. Paul, MN, USA), and the cervical puncture was manually compressed for 10 minutes. No immediate complications were noted. The patient was uneventful during an 11-month follow-up period, with a functional outcome of a modified Rankin Scale (mRS) score of 1.

\section{Case 2}

An 80-year-old male with a medical history of hypertension and hyperlipidemia had previously had a left ethmoidal dural arteriovenous fistula, which was causing intracerebral hemorrhage. He had received craniectomy and stereotactic radiosurgery 10 years before the present case. During a follow-up magnetic resonance study, severe left M1 stenosis was found (Fig. 3A). The premorbid functional outcome was an mRS score of 1. In the present case, he presented to the emergency department with severe dysarthria, aphasia, and right hemianopia for 70 minutes. The NIHSS score was 10 on arrival. CTA showed a left M1 acute occlusion (Fig. 3B). Extremely tortuous aorta and CCA were also noted (Fig. 3C). Endovascular revascularization was conducted immediately following discussion.

However, 40 minutes following femoral puncture, the GS could not be successfully inserted into a stable position in the CCA. The PBWT was applied after disinfection and preparation of the neck (Fig. 3D). The GS was successfully inserted into the distal CCA. The reperfusion catheter could not pass the carotid siphon. A microcatheter (Prowler Select 

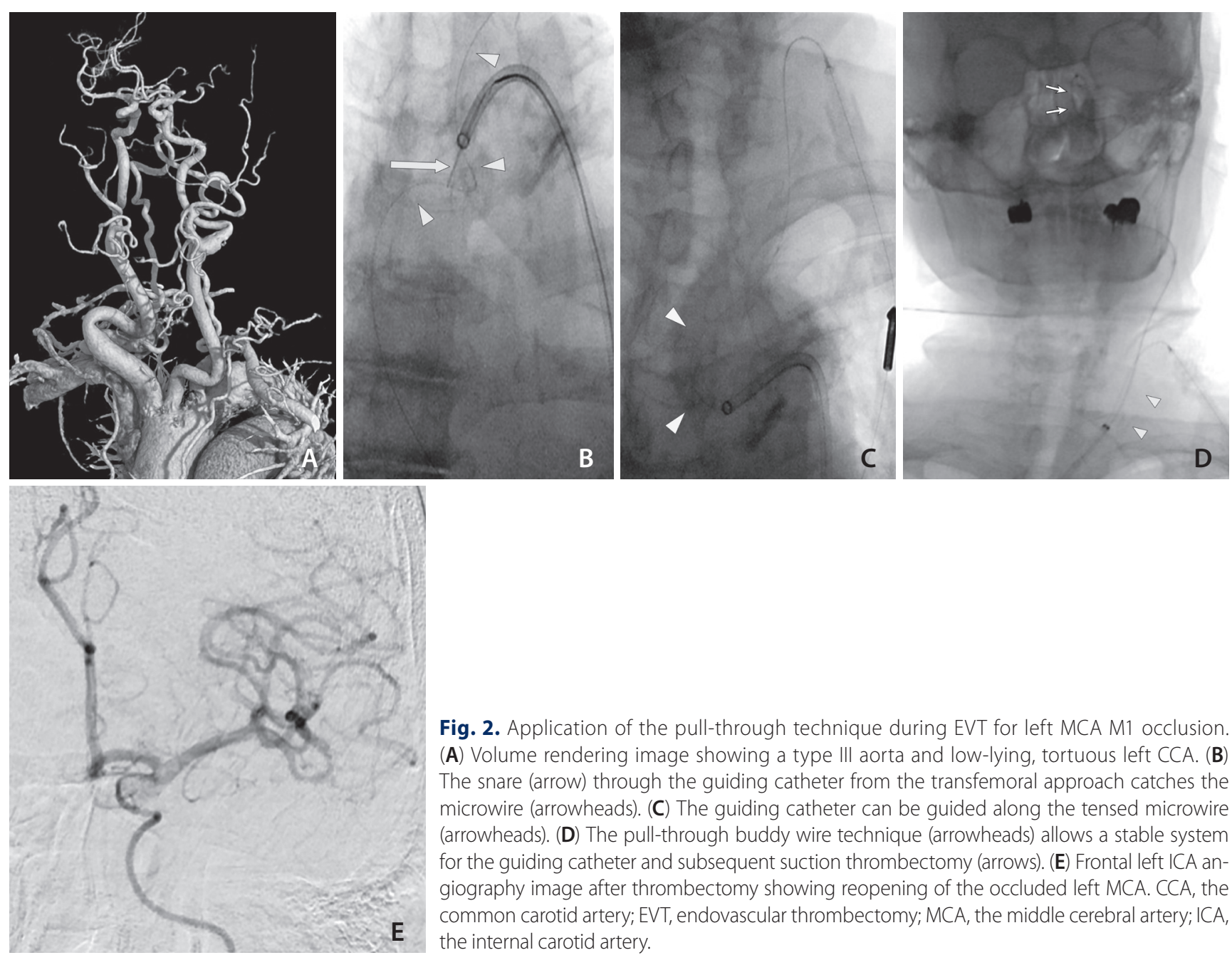

Fig. 2. Application of the pull-through technique during EVT for left MCA M1 occlusion. (A) Volume rendering image showing a type III aorta and low-lying, tortuous left CCA. (B) The snare (arrow) through the guiding catheter from the transfemoral approach catches the microwire (arrowheads). (C) The guiding catheter can be guided along the tensed microwire (arrowheads). (D) The pull-through buddy wire technique (arrowheads) allows a stable system for the guiding catheter and subsequent suction thrombectomy (arrows). (E) Frontal left ICA angiography image after thrombectomy showing reopening of the occluded left MCA. CCA, the common carotid artery; EVT, endovascular thrombectomy; MCA, the middle cerebral artery; ICA, the internal carotid artery.

Plus; Codman, Raynham, MA, USA) was navigated through the occluded MCA segment, and a salvaged stent (Enterprise; Cordis, Miami Lakes, FL, USA) was implanted (Fig. 3E). $\mathrm{TICl} 2 \mathrm{~b}$ reperfusion was achieved despite residual stenosis. The procedure time from femoral puncture to recanalization was 96 minutes. The procedural time was 54 minutes from femoral puncture to guiding catheter placement inside CCA, 10 minutes for carotid puncture, and the total time taken to complete recanalization was 96 minutes. Tirofiban infusion was initiated after intracranial stent implantation. The cervical puncture site was managed with manual compression without incident. The femoral puncture site was closed with an 8-Fr VCD.

Two weeks after discharge, the patient's symptoms were ameliorated and his NIHSS score was 6 with shuffling gait and aphasia. He received dual antiplatelet therapy. However, in-stent occlusion was noted 3 months later after an episode of acute stroke. No further endovascular treatment was performed. His mRS score was 3 at 3 months after recurrent stroke.

\section{DISCUSSION}

TCA is a commonly mentioned alternative for difficult EVT, and special care should be taken to avoid large arterial puncture wounds. In this report, the PBWT, a modified TCA technique, was demonstrated. Its advantage is its ability to minimize potential vascular trauma to the CCA while maintaining stable support of the GS.

TCA has been adapted in many neuroendovascular treatments, including arteriovenous malformation embolization, aneurysm coiling, and carotid artery stenting. ${ }^{4-7,10-12}$ An abundance of literature has been published regarding TCA 


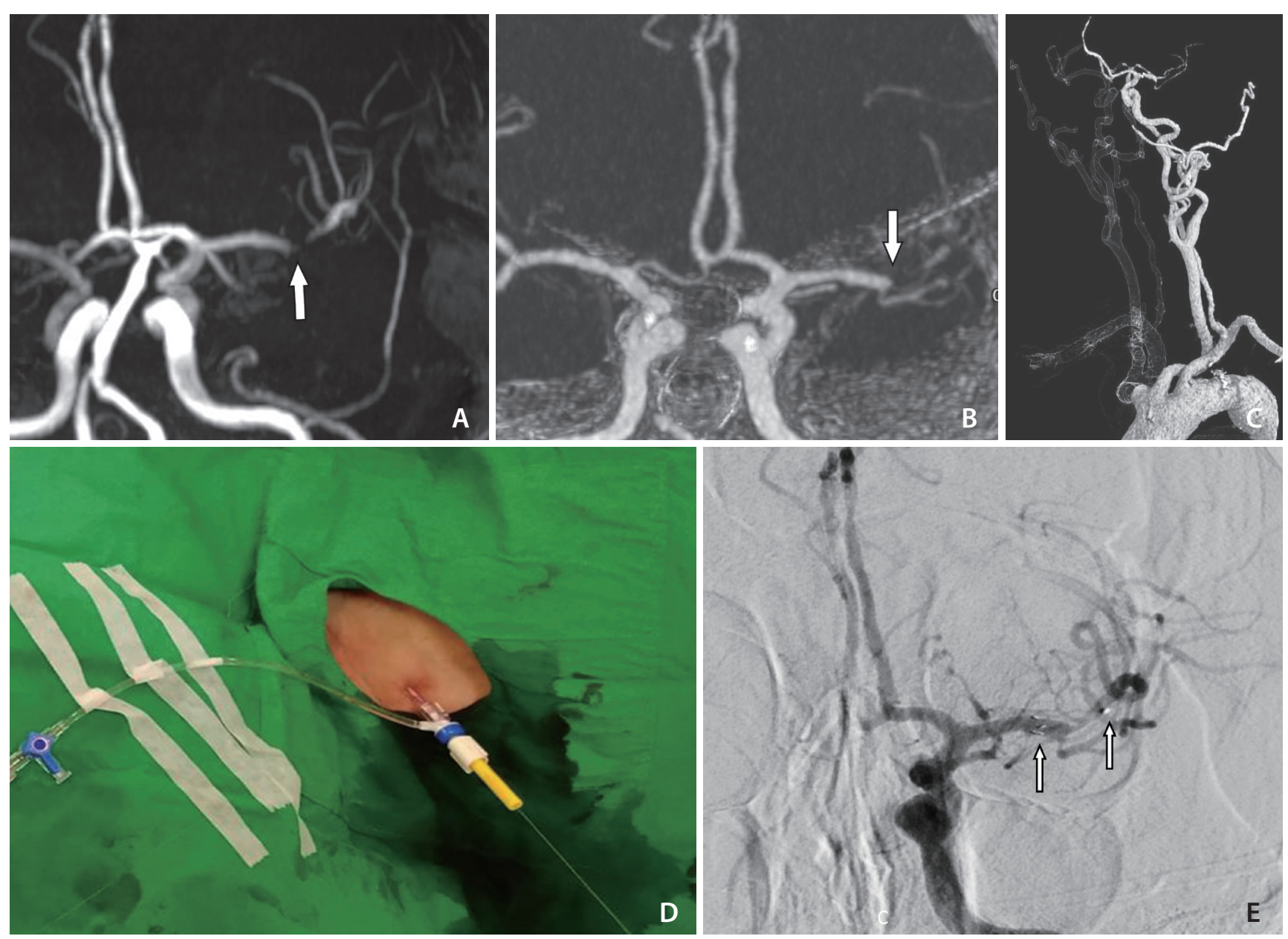

Fig. 3. Switch to the pull-through technique during stenting for acute occlusion of the left MCA M1 with underlying severe stenosis. (A) MRA findings showing underlying severe stenosis of the left MCA M1 (arrow). (B) Reformatted intracranial CTA revealing acute occlusion of the MCA (arrow). (C) Volume-rendered CTA showing a low-lying left CCA orifice with excessive tortuosity. (D) The left neck puncture site fixed with a torque device. (E) Frontal left ICA angiography image showing the recanalization of the MCA after stent placement (arrows). CTA, computed tomography angiography; CCA, common carotid artery; ICA, internal carotid artery; MCA, middle cerebral artery; MRA, magnetic resonance angiography.

in relation to $E V T^{5,6,8,10,11,13}$ Sonography-guided puncture of the cervical artery is simple and has not been reported to cause difficulty achieving access ${ }^{6}$; however, TCA with a large-bore catheter may result in complications, such as cervical artery dissection, intracranial thrombosis, and cervical hematoma. ${ }^{4,14}$ Carotid dissection by using a sheath occurred in 9 of 579 patients included in a review by Sfyroeras et al..$^{14}$. Three cases were resolved after stent graft placement, and the others were treated with surgical arteriotomy. Cervical hematoma is another possible complication of TCA. Hematoma formation in the neck can result in life-threatening airway compression. ${ }^{12}$ Moreover, regional mass effects may increase the risk of cervical neuropathy, especially in patients receiving anticoagulation, antiplatelet, or intravenous throm- bolysis treatment or when a cervical sheath larger than $6 \mathrm{Fr}$ is used. ${ }^{9,15}$ Although some researchers have reported that the application of manual compression can achieve hemostasis after TCA, the method is not always effective following EVT. For patients with difficult anatomy of the neck artery and those in a state of agitation following EVT, manual compression may be challenging. ${ }^{16}$ Commercially available VCDs are currently only approved for transfemoral interventions, and the suitability of their use for other vascular routes remains questionable. Surgical cutdown of the CCA allows for direct arterial closure and theoretically reduces local hematoma after TCA. ${ }^{17}$ Nevertheless, surgical cutdown takes more time and may require more training than direct puncture. To minimize the risk of TCA, a smaller puncture wound with a small 
catheter and microwire may help.

The pull-through technique was first reported in 1988 for occluded iliac artery treatment. ${ }^{18}$ The technique can provide a steady force for the wire and catheter passing through tortuous vessels, allowing the procedure to be performed smoothly. Because it provides a stable wire tension, it is widely used in peripheral interventions. ${ }^{19}$ He et al. ${ }^{20}$ found that the pull-through technique provided better catheter support in the treatment of high-flow hemodialysis arteriovenous fistula and during stenosis angioplasty, with shorter procedure times. No significant complications in postprocedure hemostasis were noted, despite the use of a guiding catheter with a relatively large diameter. ${ }^{19,20}$ The technique has been described for treating vascular stenosis through angioplasty and stenting. ${ }^{18-20}$ To our knowledge, the use of the pull-through technique has not yet been reported for acute stroke intervention.

The most notable advantage of the PBWT is that it reduces the puncture wound size in the CCA. In our experience, manual compression for 10 minutes is sufficient for achieving hemostasis. The second advantage is that the locked microwire can stabilize the GS during the following procedure. However, there are some potential drawbacks to bear in mind. First, the reperfusion catheter is contained in the GS parallel to the microwire; therefore, a larger GS should be used. We used an 8-Fr GS instead of the more common 6-Fr GS. Second, the tip location of the GS was limited by the puncture site at the distal CCA. Although the system was stable, the low position of the GS could not further reduce redundancy of the internal carotid artery. Third, snaring the microwire in the aortic arch is not always easy and may require additional time if operators are unfamiliar with the technique.

\section{CONCLUSION}

The PBWT might be feasible for modified TCA that can be applied in EVT to overcome excessive tortuosity. It can have the potential benefit of decreased risk of cervical puncture site complications.

\section{Fund}

None.

\section{Ethics Statement}

The study was approved by the research ethics committee of the National Taiwan University Hospital.

\section{Conflicts of Interest}

The authors have no conflicts to disclose.

\section{Author Contributions}

Concept and design: CWL. Analysis and interpretation: YHL and YCH. Data collection: PYC and YCH. Writing the article: PYC. Critical revision of the article: YHL. Statistical analysis: PYC and YCH. Overall responsibility: CWL.

\section{ORCID}

Pin-Yi Chiang: https://orcid.org/0000-0002-8701-8347

Yen-Heng Lin: https://orcid.org/0000-0003-4327-7432

Yu-Cheng Huang: https://orcid.org/0000-0003-0605-3085

Chung-Wei Lee: https://orcid.org/0000-0002-3573-2506

\section{REFERENCES}

1. Powers WJ, Rabinstein AA, Ackerson T, Adeoye OM, Bambakidis NC, Becker K, et al. Guidelines for the early management of patients with acute ischemic stroke: 2019 update to the 2018 guidelines for the early management of acute ischemic stroke: a guideline for healthcare professionals from the American Heart Association/American Stroke Association. Stroke 2019;50:e344-e418

2. Goyal M, Menon BK, van Zwam WH, Dippel DW, Mitchell PJ, Demchuk AM, et al.; HERMES collaborators. Endovascular thrombectomy after large-vessel ischaemic stroke: a meta-analysis of individual patient data from five randomised trials. Lancet 2016;387:1723-1731

3. Leischner H, Flottmann F, Hanning U, Broocks G, Faizy TD, Deb-Chatterji $M$, et al. Reasons for failed endovascular recanalization attempts in stroke patients. J Neurointerv Surg 2019;11:439-442

4. Ribo M, Flores A, Rubiera M, Pagola J, Mendonca N, Rodriguez-Luna D, et al. Difficult catheter access to the occluded vessel during endovascular treatment of acute ischemic stroke is associated with worse clinical outcome. J Neurointerv Surg 2013;5 Suppl 1:i70-i73

5. Styczen H, Behme D, Hesse AC, Psychogios MN. Alternative transcarotid approach for endovascular treatment of acute ischemic stroke patients: a case series. Neurointervention 2019;14:131-136

6. Jadhav AP, Ribo M, Grandhi R, Linares G, Aghaebrahim A, Jovin 
TG, et al. Transcervical access in acute ischemic stroke. J Neurointerv Surg 2014;6:652-657

7. Colombo E, Rinaldo L, Lanzino G. Direct carotid puncture in acute ischaemic stroke intervention. Stroke Vasc Neurol 2020;5:71-79

8. Wiesmann M, Kalder J, Reich A, Brockmann MA, Othman A, Greiner $A$, et al. Feasibility of combined surgical and endovascular carotid access for interventional treatment of ischemic stroke. J Neurointerv Surg 2016;8:571-575

9. Blanc R, Piotin M, Mounayer C, Spelle L, Moret J. Direct cervical arterial access for intracranial endovascular treatment. Neuroradiology 2006;48:925-929

10. Mokin M, Snyder KV, Levy El, Hopkins LN, Siddiqui AH. Direct carotid artery puncture access for endovascular treatment of acute ischemic stroke: technical aspects, advantages, and limitations. J Neurointerv Surg 2015;7:108-113

11. Matsumoto H, Fujita K, Miki J, Tsuji N, Terada T, Itakura T. [Direct carotid approach for carotid angioplasty and stenting with a small incision: a technical case report]. No Shinkei Geka 2004;32:735-740 Japanese

12. Nii K, Kazekawa K, Onizuka M, Aikawa H, Tsutsumi M, Tomokiyo $M$, et al. Direct carotid puncture for the endovascular treatment of anterior circulation aneurysms. AJNR Am J Neuroradiol 2006;27:1502-1504

13. Vidale S, Longoni M, Valvassori L, Agostoni E. Mechanical thrombectomy in strokes with large-vessel occlusion beyond 6 hours: a pooled analysis of randomized trials. J Clin Neurol 2018;14:407412
14. Sfyroeras GS, Moulakakis KG, Markatis F, Antonopoulos CN, Antoniou GA, Kakisis JD, et al. Results of carotid artery stenting with transcervical access. J Vasc Surg 2013;58:1402-1407

15. Gandhi CD, Al Mufti F, Singh IP, Abruzzo T, Albani B, Ansari SA, et al.; Standards and Guidelines committee of the Society of Neurolnterventional Surgery (SNIS). Neuroendovascular management of emergent large vessel occlusion: update on the technical aspects and standards of practice by the Standards and Guidelines Committee of the Society of Neurolnterventional Surgery. J Neurointerv Surg 2018;10:315-320

16. Fjetland L, Roy S. Transcarotid endovascular thrombectomy for acute ischemic stroke. J Vasc Interv Radiol 2018;29:1006-1010

17. Dorfer C, Standhardt H, Gruber A, Ferraz-Leite H, Knosp E, Bavinzski G. Direct percutaneous puncture approach versus surgical cutdown technique for intracranial neuroendovascular procedures: technical aspects. World Neurosurg 2012;77:192200

18. Loose HW, Ryall CJ. Common iliac artery occlusion: treatment with pull-through angioplasty. Radiology 1988;168:273-274

19. Mitsuhashi Y, Nishio A, Kawakami T, Shibamoto K, Yamagata T, Ichinose T, et al. New pull-through technique using the superficial temporal artery for transbrachial carotid artery stenting. Neurol Med Chir (Tokyo) 2009:49:320-324

20. He Q, Yu B, Shi W, Tan J, Zhu L, Liang K. Pull-through technique combined with percutaneous angioplasty for treating highgrade arteriovenous fistula stenosis. J Vasc Access 2020;21:223229 